\title{
PHASE TRANSITIONS DURING INFLATION AND CHEMICALLY INHOMOGENEOUS UNIVERSE
}

\author{
A.D. Dolgov [ \\ INFN section of Ferrara \\ Via del Paradiso 12, 44100 Ferrara, Italy
}

\begin{abstract}
Several models of baryo(lepto)- genesis that give rise to large inhomogeneities in the composition of the universe are presented. In particular, a variation of primordial abundances of light elements by the factor 2-5 at large distances is predicted. A cosmological model of baryonic island is considered. Creation of domains with very large baryon and antibaryon number is described. Such domains mostly collapsed into primordial black holes that could be the dominant part of (non-standard) cosmological cold dark matter with a widely dispersed mass spectrum. A non-collapsed part of these bubbles might make clouds of matter or antimatter with an enriched abundances of metals. A mechanism for creation of such exotic objects can be realized by mixed order phase transitions induced by the inflaton field.
\end{abstract}

\section{Introduction}

It is commonly believed that the universe is the same everywhere (at least inside the present day horizon). This is the well known Copernicus or cosmological principle, or the principle of cosmic democracy. Observational astronomical data quite well agree with this principle and especially strong argument comes from the perfect angular smoothness of the cosmic microwave background radiation (CMBR). However, chemical inhomogeneities may escape detection by CMBR either because they do not necessarily imply inhomogeneities in mass or energy densities or if their size is too small to be observable

\footnotetext{
${ }^{1}$ Also: ITEP, Bol. Cheremushkinskaya 25, Moscow 113259, Russia.
} 
by the contemporary CMBR telescopes. A detection of these objects by other astronomical methods may be inhibited by their large distance from the Earth. Thus at the present level of our knowledge, there seems to be plenty of room for "pieces" of universe with an exotic matter content. Of course the hypothesis of cosmologically large chemical inhomogeneities looks quite striking but it already existed in different forms for quite a long time (e.g. the idea of domains of cosmic antimatter). Several simple theoretical models predict such phenomena. The universe with non-homogeneous chemistry can be created if certain phase transitions occurred at inflationary stage leaving behind astronomically large bubbles with different physical conditions. Such phase transitions should have a rather unusual character. The effective mass of a scalar (Higgs-like) field might be negative but only during a finite (and rather small) period of time. This period should take place not too long before the end of inflation. These properties could be realized by a simple coupling of this Higgs-like field (order parameter) to the inflaton. The models with this property predict formation of chemically different domains in the early universe that might be astronomically large but not too large and sufficiently rare to escape existing observational bounds.

Since this is the last school on cosmological phase transitions of this century and even of the Second Millennium it is tempting to speculate about cosmology in the coming XXI century. To verify or to disprove Copernicus principle seems to be a great challenge for the future (hopefully not too distant one).

The content of the lecture is the following. First, we discuss the model of baryo(lepto)genesis that could give rise to bubbles of chemically different phases in the universe. In sec. 3 we present a model of inhomogeneous lepto-genesis that predicts an existence of cosmologically large regions with strongly varying primordial abundances of light elements. The simplest version of the model predicts $2 / 3$ of the sky with the so called normal abundances (25\% of $H e^{4}$ and about $3 \cdot 10^{-5}$ of deuterium), 1/6 of the sky with much richer fraction of light elements ( $50 \%$ of $H e^{4}$ and about $15 \cdot 10^{-5}$ of $D$ ), and $1 / 6$ of 
the sky with a poor production of light elements ( $12 \%$ of $H e^{4}$ and about $1.5 \cdot 10^{-5}$ of $\left.D\right)$. In the next section an inhomogeneous baryogenesis leading to formation of baryonic and anti-baryonic cosmological islands is discussed. After that we consider a possible mechanisms of creation of cosmic antimatter in the universe dominated by baryons. Related to that is a model of formation of primordial black holes that could deliver a rather unusual form of cold dark matter with a very broad mass spectrum of constituent "particles".

\section{Baryo- and Lepto- Genesis}

After Sakharov [1] formulated three general principles of baryogenesis: non-conservation of baryonic charge, breaking of particle-antiparticle symmetry, and deviation from thermal equilibrium, a variety of models were invented that predict, in particular, noticeable inhomogeneities in baryon or lepton number densities (isocurvature perturbations) which might lead to chemically inhomogeneous universe (for a review see ref. [2]). We will describe here one of the models that is especially interesting from this point of view, namely the Affleck and Dine scenario [3]. The model is based on a supersymmetric theory that possesses the following generic properties. First, there exist scalar fields with non-zero baryonic and/or leptonic charges. We denote these scalar baryons or leptons generically as $\chi$. Such fields are obligatory in any supersymmetric model. For example they could be super-partners of quarks and leptons. Second, the potential of these fields $\chi$ have the so called flat directions along which the energy does not rise. Such flat direction might exist, in particular, prior to spontaneous symmetry breaking before particle masses were generated by the Higgs mechanism.

During inflation scalar fields (in contrast to fermions) may form a condensate by rising quantum fluctuations along flat directions [4, 5, 6, 7]. A large baryonic charge can be stored in this condensate if baryon number is not conserved. The subsequent decay

of this condensate in the course of processes that conserve baryonic charge would create a cosmological baryon (or lepton) asymmetry that might reach very large values, even 
close to unity. Recall that the observed value is

$$
\beta=N_{B} / N_{\gamma} \approx(3-5) \cdot 10^{-10}
$$

The model permits to create simultaneously a small baryon asymmetry in agreement with observations together with a large lepton asymmetry [8, 2, 9]. Moreover the asymmetries may be inhomogeneous and the scale of variation of lepton asymmetry may be much smaller then the scale of variation of baryon asymmetry [8, 2].

To illustrate the main features of this scenario let us consider the toy model with the following potential term:

$$
U(\chi)=m^{2}|\chi|^{2}+\lambda_{1}|\chi|^{4}+\lambda_{2}\left(\chi^{4}+\chi^{4}\right)
$$

In the FRW cosmology, where the metric is given by $d s^{2}=d t^{2}-a^{2}(t) d r^{2}$, the equation of motion for $\chi$ has the form:

$$
\left(\partial_{t}^{2}-\partial_{k}^{2} / a^{2}\right) \chi+3 H \dot{\chi}+U^{\prime}(\chi)=0
$$

where $H=\dot{a} / a$ is the Hubble parameter.

If $\lambda_{2}=\lambda_{1} / 2$ the potential has flat directions in the limit $m=0$ and the field could easily evolve along them. It is convenient to introduce phase and absolute value of the field:

$$
\chi=r e^{i \theta}
$$

and in terms of this quantities flat directions go along $1+\cos 4 \theta=0$.

The field $\chi$ may possess leptonic or baryonic charge (or a mixture of both) and the corresponding vector current is given by

$$
j_{\mu}=i\left(\chi^{*} \partial_{\mu} \chi-\partial_{\mu} \chi^{*} \chi\right)
$$

According to the equation of motion (3) the current is not conserved if $\lambda_{2} \neq 0$ :

$$
\partial_{\mu} j^{\mu}=2 i \lambda_{2}\left[\chi^{4}-\left(\chi^{*}\right)^{4}\right]
$$


In the homogeneous case the equation of motion takes the form:

$$
\ddot{\chi}+3 H \dot{\chi}+U^{\prime}(\chi)=0
$$

It is the Newtonian equation of motion for a point-like body in two dimensional space of the complex plane $\chi$ in the potential $U(\chi)$. Here $\ddot{\chi}$ is the acceleration, $U^{\prime}$ is the force, and $3 H \dot{\chi}$ is the "liquid" friction term. Baryonic charge density is given by:

$$
B(t) \equiv j_{0}=r^{2} \dot{\theta}
$$

and, in terms of the mechanical analogy, coincides with the angular momentum of the body moving in the potential $U$. If the potential is spherically symmetric, i.e. it depends only on $r=|\chi|$ the angular momentum (or baryonic charge) is conserved. The presence of valleys breaks the symmetry and accordingly the conservation of $j_{0}$.

During inflation, when/if $m \ll H$, the field $\chi$ is infrared unstable and its quantum fluctuations rise in accordance with the law [5, 6, 7]:

$$
\left\langle\chi^{2}\right\rangle=\frac{H^{3} t}{2 \pi} \rightarrow \min \left\{\left(\sim H^{4} / m^{2}\right),\left(\sim H^{2} / \sqrt{\lambda}\right)\right\}
$$

Hence the field $\chi$ might acquire a large expectation value along flat directions and when inflation is over and a small slope of the potential created by $m^{2}|\chi|^{2}$ becomes essential, the field returns to the origin releasing baryonic (or leptonic) charge by the decay into quarks or leptons. However, it is important to keep in mind that if the field evolved only in radial direction so that $\dot{\theta}=0$, the baryonic charge accumulated by quantum fluctuations would be zero, as is seen from eq. (8). Hence a motion in orthogonal direction is necessary for efficient baryogenesis. This motion may be produced by quantum fluctuations across the valley. So when the field $\chi$ "lives" in the valley, far from the origin, it would oscillate between steep walls of the valley slowly approaching zero. When the field comes sufficiently close to the origin the quartic terms in the potential become negligible and the field evolution is governed by the quadratic term $m^{2}|\chi|^{2}$. In this region the field rotates around the origin with a decreasing amplitude. 
The damping of oscillation both in the valleys and near origin is forced by the following two phenomena: by the Hubble friction that diminishes the amplitude according to $|\chi| \sim a^{-3 / 2}$ and by the particle production which is strongly model dependent and might be very much different for baryonic and leptonic $\chi$ 's. Estimates of the particle production rate can be found in ref. [2]. This effect may strongly damp motion along orthogonal direction in the valleys and significantly diminish the generation of the charge asymmetry. The decay of $\chi$ at this stage creates average zero baryonic charge because the angular velocity $\dot{\theta}$ frequently changes sign. On the other hand, the decay of the field on the final stage, when it rotates around the origin, proceeded with charge conservation and the produced quarks or leptons would have baryonic or leptonic charge equal to the initial magnitude of the angular momentum of $\chi$, at the moment when $\chi$ arrived to the flat region and oscillations in angular direction changed into rotation. The magnitude of the latter is determined by the damping in the valley, while direction of rotation (i.e. the sign of baryon or lepton asymmetry) is determined stochastically by the direction of quantum fluctuations across the valley during inflation. In such a model $\mathrm{C}$ and $\mathrm{CP}$ non-conservation is not necessary. The latter is created by chaotic initial conditions. The size of domains with a certain sign of the charge asymmetry may be very large due to exponential expansion. However, a concrete realization of the model can meet serious problems and some fine-tuning of parameters or initial conditions may be necessary.

The asymmetry generated by the decay of $\chi$ at the final stage could be very large if the life-time of $\chi$ with respect to decay (or to the particle production rate) is sufficiently long. The oscillating massive field has the non-relativistic equation of state, $P=0$ (here $P$ is the pressure density), so the energy density of such field decreases as $1 / a^{3}$. It is one power of $a$ slower than the decrease of the energy density of relativistic matter. The slower red-shift may create the situation when the matter in the universe is completely dominated by baryons (or leptons) with a negligible amount of antiparticles.

If $\mathrm{C}(\mathrm{CP})$ violation is explicit then the picture may be somewhat different. In our toy 
model we can realize this case assuming that coupling constant $\lambda$ and mass are complex, so that the potential takes the form:

$$
U(\chi)=m^{2} \chi^{2}+\left(m^{*}\right)^{2}\left(\chi^{*}\right)^{2}+\lambda \chi^{4}+\lambda^{*}\left(\chi^{*}\right)^{4}
$$

Both terms are necessary, otherwise the CP-odd phase can be rotated away. In this potential the field $\chi(t)$ may acquire a non-vanishing angular momentum after it traveled down along the bottom of the valley without any orthogonal motion and closer to the origin it changes "rails" from the lambda-valley to the mass-valley that has a different direction. The transition from the bottom of one valley to another creates a transverse motion or, in other words, a nonzero baryonic or leptonic charge.

\section{$3 \quad$ Large and Inhomogeneous Lepton Asymmetry}

Models based on the picture described in the previous section allow to obtain a small baryonic and a large leptonic asymmetry if e.g. the decay of the baryonic $\chi$-field is faster than the decay of the leptonic one. In this case baryonic angular fluctuations in the valleys would be stronger suppressed than leptonic ones, so the initial value of the baryonic charge at the beginning of rotation would be smaller than the initial value of the leptonic charge. Moreover, the dilution of the produced baryons by expansion could be stronger than the dilution of later created leptons.

The characteristic wave length of the variation of the baryon $\left(l_{B}\right)$ or lepton $\left(l_{L}\right)$ charge asymmetry was estimated in ref. [2] and is approximately equal to the smaller of $H_{i}^{-1} \exp (1 / \sqrt{\lambda})$ and $H_{i}^{-1} \exp \left[\left(H_{i} / m\right)^{2}\right]$. In particular, it is possible to create the universe with a small and homogeneous baryon asymmetry and large (of the order of unity) lepton asymmetry, which in addition may be inhomogeneous at the scales well inside the present day horizon [8, 10, 11]. Such domains of leptonic charge would create very strong density inhomogeneities and, in turn, too large angular fluctuations of CMBR. However the flavor symmetry between different lepton families, $e \leftrightarrow \mu \leftrightarrow \tau$, permits to 
avoid this problem because in this case one would expect domains that are symmetric with respect to permutation of different leptonic charges and in first approximation the density contrast is vanishing [10, 11].

In the simplest version of this scenario the universe would be filled with equally probable domains with $\left(L_{e}, L_{\mu}, L_{\tau}\right)=( \pm 1,0,0)$ and with all possible permutations between leptonic charges. Evidently there are no walls between such domains, as follows from the way of their creation, and so the cosmological problem of heavy domain walls [12] is avoided. Since the abundances of light elements produced at big bang nucleosynthesis are especially sensitive to charge asymmetry of electronic neutrinos, there would be two types of anomalous domains with low and high abundances and 4 types of the normal ones with the same normal abundances, as is written in the introduction.

As discussed in the papers [10, 11] the characteristic distances between the domains should be larger than a few hundred Mpc to agree with the observed small angular fluctuations of the CMBR temperature, $\delta T / T$. Another possibility of small size domains, that could create a large $\delta T / T$ on very small scales, which are allowed by observations, was not explored but possibly, though not surely, this option is excluded by direct observation of the light element abundances. Still it remains surprising that twice larger mass fraction of $\mathrm{He}^{4}$ at not so large distances is not yet observationally excluded. An-

other signature of such inhomogeneities in primordial $\mathrm{He}^{4}$ is a strong variation of the exponential slope in $\delta T / T$ at small angles (at $l>10^{3}$ ) at different patches on the sky. Possibly it is the most promising way to obtain an upper limit on inhomogeneities of primordial $H e^{4}$.

\section{Inflaton Induced Phase Transitions and Baryonic Islands}

If the field $\phi$ that is responsible for baryo(lepto)-genesis is coupled to inflaton then a very interesting pattern of charge asymmetries may be created. This field $\phi$ could be 
e.g. the Affleck-Dine one described in the previous section or the complex scalar field whose condensate creates spontaneous breaking of charge symmetry [13]. The coupling to inflaton, $\Phi$, is assumed to be of the general renormalizable form:

$$
\mathcal{L}_{\text {int }}=\lambda|\phi|^{2} \Phi^{2}+g|\phi|^{2} \Phi=\lambda\left(\Phi-\Phi_{1}\right)^{2}|\phi|^{2}-\lambda \Phi_{1}^{2}|\phi|^{2}
$$

where $\Phi_{1}=g / 2 \lambda$ is a constant. We assumed that the interaction conserves $\mathrm{C}(\mathrm{CP})$, though it is not necessary.

Generically the effective mass of the field $\phi$ may contain the following contributions:

$$
\left(m_{e f f}^{\phi}\right)^{2}=m_{0}^{2}+\xi R+\beta T^{2}+\lambda\left(\Phi-\Phi_{1}\right)^{2}
$$

where $R$ is the curvature scalar and the correspoding term comes from a possible nonminimal coupling to gravity. The third term comes from the interaction with thermal bath, and the last one comes from the interaction with inflaton (11). Note that at inflationary stage $R=12 H^{2}$, while at radiation dominated stage $R=0$. With such a form of mass term one can easily visualize the following picture. At an early inflationary stage the effective mass squared of $\phi$ is large and positive, so that $\phi$ sits near the origin $\phi=0$. When the inflaton evolves down and approaches the value $\Phi \approx \Phi_{1}$ the mass squared becomes negative and remains such for some period of time determined by the inflaton evolution. When the amplitude of the inflaton tends to zero and inflation ends, $m_{\text {eff }}^{2}$ may become positive again. During a relatively short period of negative $m^{2}$ the road is open for $\phi$ to travel away from the origin and its subsequent destiny depends upon its self-interaction potential. The field may either return to the origin, or stay for a while in a false vacuum state with $\langle\phi\rangle \neq 0$, or evolve far away along one or other flat directions of the potential. Correspondingly the generation of baryon (or lepton) asymmetry would be quite different. Some striking possibilities are discussed in the papers [14, 15, 16] or in the review [2].

In particular, our universe may be a huge baryonic island floating in the sea of dark matter [14, 15]. This can be achieved if the field $\phi$ is responsible for the spontaneous 
breaking of $\mathrm{C}$ and $\mathrm{CP}$ symmetries [13], so that cosmological charge asymmetry might be generated only in the regions where $\langle\phi\rangle \neq 0$. The size of this island may be of the order of the present day horizon or much larger. In the first case a small observed dipole asymmetry of CMBR forces us to live very close to the center of the island and it makes the model rather unnatural. Its interesting property is that the angular distribution of the CMBR temperature in this model would have an intrinsic dipole moment:

$$
D=\left(\frac{\delta T}{T}\right)_{\text {dipole }} \sim\left(\frac{d}{R_{i}}\right)
$$

where $R_{i}$ is the radius of the island and $d$ is our displacement from the Center of the world. In this case the dipole contribution into angular fluctuations of CMBR is partly given by our peculiar motion and partly by the displacement from the Center and one does not need the very large value of the velocity, about $600 \mathrm{~km} / \mathrm{sec}$, whose origin is mysterious, to explain the observed dipole. There should also be an intrinsic quadrupole with a rather large value $Q \sim\left(d / R_{i}\right)^{2}$, which is close to the observed one.

The size of the island may be of super-horizon scale but nevertheless the angular fluctuations imprinted on CMBR by the existence of the boundary could be observable [21]. Of course their magnitude would be much smaller than in the sub-horizon case and we may live far from the Center without breaking the observational limits. Still the large magnitude of the dipole asymmetry of CMBR may be explained by the insular hypothesis.

Models of baryogenesis with spontaneous breaking of charge symmetry predict formation of cosmological matter-antimatter domains, as was noticed in ref. [17]. The size of these domains may be cosmologically large if after their formation the universe passed through a period of exponential expansion (inflation) [18. A review of the earlier ideas on the subject can be found in the paper [19] or in the recent one [20], which contains a discussion and the list of references to later development. In the model described above $\mathrm{C}(\mathrm{CP})$ is broken spontaneously but only in finite bubbles, while outside the bubbles particles and antiparticles are completely symmetric and cosmological charge asymmetry 
in these regions is vanishing. As in any model with spontaneous breaking of charge invariance the universe as a whole should be charge symmetric, so there must be an equal number of bubbles and anti-bubbles. We assumed above that the size of the bubbles (or at least of our bubble) is very large, close to the horizon or much larger than it. In such a model other bubbles most probably should be far beyond the horizon and be unobservable.

In principle another scenario is possible when the bubbles are relatively small so that there are many of them inside the horizon and the universe reminds a piece of Swiss cheese where bubbles of matter and antimatter are separated by voids filled with dark matter. There is no direct contact between them and thus no $p \bar{p}$-annihilation. This weakens the limits on antimatter domains found in refs. [22, 23] but the observed distribution of baryonic (luminous) matter seems to disagree with such a picture. Moreover, angular variation of the CMBR temperature induced by such isocurvature perturbations are likely to be unacceptably large. A related analysis was performed in references [24, 25] but the case considered there rather differs from this model and some more work is desirable.

\section{Large Baryon Asymmetry, Primordial Black Holes, and Dark Matter}

In the model of the previous section the magnitude of baryon asymmetry inside the islands was normal (1), but the sign could be different. The sea outside the islands was charge symmetric. Here we will consider another exotic scenario of baryogenesis according to which the average baryon asymmetry is normal and positive but in its background there could be relatively small (but still astronomically large) bubbles with a very large (even close to unity) asymmetry. Such a picture can be realized with the help of the scalar baryon discussed in sec. 2 if the flat directions of its potential are "locked" by a positive value of $m_{\text {eff }}^{2}$ during almost all inflationary stage and the window 
is only open when the inflaton field $\Phi$ is close to $\Phi_{1}$, see eq. (12). In such a scenario the Affleck-Dine field $\chi$ may reach large values and accumulate a big baryonic charge only in relatively small bubbles, where the field succeeded to reach a certain critical value. This process is stochastic and its probability may be sufficiently small so only a minor fraction of the universe would be occupied by this baryon rich bubbles (see ref. [16] for the details). If the potential of $\phi$ is $\mathrm{C}(\mathrm{CP})$-symmetric then the probability of baryon rich and antibaryon rich bubbles would be the same. So according to this scenario the cosmological background is baryo-asymmetric and each bubble is (strongly) baryoasymmetric but on the average there is equal amount of baryonic and anti-baryonic bubbles.

The subsequent destiny of these baryon rich bubbles depends upon their size and the value of the baryon asymmetry. Predominantly they would end as primordial black holes formed at the stage when baryons became nonrelativistic. According to calculations of ref. 16] the mass spectrum of the black holes in a simple version of the model is given by the log-normal distribution:

$$
\frac{d N}{d M}=C \exp \left(-\gamma \ln ^{2} \frac{M}{M_{1}}\right)
$$

where $C, \gamma$, and $M_{1}$ are constants expressed through some unknown parameters of the underlying theory. We cannot make any reliable prediction about their value. They may easily vary by several orders of magnitude. So in what follows we will treat them as free parameters to be constrained from observations.

According to this model not only the bubble sizes are stochastically distributed but also the magnitude of baryon asymmetry inside them. Depending upon the values of these two parameters the bubbles would either form black holes and whether they are baryonic or anti-baryonic could not be distinguished, because black holes do not have baryonic hairs. Smaller size objects or those with a smaller value of the asymmetry do not collapse and on the tail of the distribution we may expect to observe clouds of antimatter in close vicinity, anti-stars, or even possibly small anti-galaxies. 
Another interesting feature of this baryon (or antibaryon) rich regions is that their primordial chemistry should be very much different from the standard one. The outcome of light elements produced during "the first three minutes" strongly depends upon the baryon-to-photon ratio, $N_{B} / N_{\gamma}$. Normally it is very small, eq. (1), and the nucleosynthesis effectively stops at $H e^{4}$. The amount of the next stable light element $L i$ is roughly 9 orders of magnitude smaller. In the case of a larger ratio $N_{B} / N_{\gamma}$ the amount of heavier elements could be much larger and the baryon rich bubbles might have much more evolved chemistry which is more typical for the system contaminated by stellar processes.

The described here mechanism easily explains an early production of quasars, while in the standard theory of structure formation this creates a serious problem. The model also explains an evolved chemistry around quasars. This could be the matter from baryon rich region that did not completely disappeared in the black hole (quasar) and which was enriched by heavier elements from the "very beginning", i.e. from big bang nucleosynthesis. In the standard approach it is assumed that heavier elements observed in the vicinity of quasars were produced as a result of stellar processes in the stars of first generation. But this is very difficult to accomodate to the standard theory of structure formation according to which structure are formed much later than the observed distant quasars.

An interesting implication of the model of primordial black hole formation with the mass spectrum (14) is that they might make a new form of cold dark matter. In contrast to the usual type of dark matter, when all dark matter particles have equal masses, this model predicts a mass dispersed CDM where particles of different masses play different role. The parameters describing the spectrum can be fixed from the observations. In particular, one could request that there is one super-heavy black hole $\left(M>10^{6} M_{\odot}\right)$ per large galaxy. Here $M_{\odot}$ is the solar mass. These super-heavy black holes can serve as seeds for galaxy formation. The bulk of dark matter may be presented by black holes with 
the masses near $10-100 M_{\odot}$, while those with the mass about $0.5 M_{\odot}$ may explain the MACHO's observations [26, 27]. In addition the model gives an explanation of formation of heavy $\left(M>100 M_{\odot}\right)$ black holes observed in galaxies. A possible simple fit: $\gamma=0.14$ and $M_{1}=0.5 M_{\odot}$, gives for the relative contribution to the universe mass density in different mass intervals the following results: $F(M<0.1)=0.3 \%, F(0.1-2)=12 \%$, $F(M<10)=38 \%, F(M=2-30)=48.5 \%, F(M=30-1000)=37.5 \%, F(M=$

$\left.10^{3}-10^{6}\right)=1.6 \%, F\left(M=10^{6}-10^{8}\right)=3.6 \cdot 10^{-9}$, where $M$ is expressed in solar mass units. Of course a more accurate analysis with the account of existing astronomical bounds on the cosmic density of black holes in different mass intervals is necessary to make more definitive conclusion [28] and numerical simulation of structure formation in CDM model with the mass spectrum of dark matter particles given by eq. (14) is desirable.

\section{References}

[1] A.D. Sakharov, Pis'ma Zh. Eksp. Teor. Fiz. 5 (1967) 32.

[2] A.D. Dolgov, Phys. Repts. 222 (1992) No. 6.

[3] I. Affleck and M. Dine, Nucl. Phys. B249 (1985) 361.

[4] T.S. Bunch and P.C.W. Davies, Proc. Roy. Soc.(London), A360 (1978) 117.

[5] A. Vilenkin and L. Ford, Phys. Rev. D26 (1982) 1231.

[6] A.D. Linde, Phys. Lett. 116B (1982) 335.

[7] A.A. Starobinsky, Phys. Lett. 117B (1982) 175.

[8] A.D. Dolgov and D.P. Kirilova, J. Moscow Phys. Soc. 1 (1991) 217.

[9] J.A. Casas, Wai Yan Cheng, G. B. Gelmini, Nucl.Phys. B538 (1999) 297. 
[10] A.D. Dolgov and B.E.J. Pagel, New Astron. 4 (1999) 223.

[11] A.D. Dolgov, "Particle Physics and the Early Universe (COSMO-98)", p.259; ed. by David O. Caldwell, American Institute of Physics.

[12] Ya.B. Zel'dovich, I.Yu. Kobzarev, and L.B. Okun, ZhETF, 67 (1974) 3.

[13] T.D. Lee, Phys. Rev. D8 (1973) 1226.

[14] A.D.Dolgov and N.S.Kardashev, Space research institute preprint-1190, 1986.

[15] A.D. Dolgov, A.F. Illarionov, I.D. Novikov and N.S. Kardashev, ZhETF, 94 (1987) 1.

[16] A. Dolgov and J. Silk, Phys. Rev. D47 (1993) 4244.

[17] R.W. Brown and F.W. Stecker, Phys. Rev. Lett. 43 (1979) 315.

[18] K. Sato, Phys. Lett. 99B (1981) 66.

[19] F.W. Stecker, Nucl. Phys. B252 (1985) 25.

[20] A.D. Dolgov, Invited Talk at EuroConference on Frontiers in Particle Astrophysics and Cosmology, San Feliu de Guixols, Spain, 30 September - 5 October 2000 (to be published in the Proceedings); hep-ph/0012107.

[21] L.P. Grishchuk and Ya.B. Zeldovich, Astron. Zh. 55 (1978) 209 [Sov. Astron. 22 (1978) 125].

[22] G. Steigman, Annu. Rev. Astr. Ap. 14 (1976) 339.

[23] A.G. Cohen, A. De Rujula, and S.L. Glashow, Astrophys.J. 495 (1998) 539;

A. De Rujula, astro-ph/9705045. Based on a talk at the Jan. 1998 Moriond Meeting at Les Arcs, France. 
[24] W.H. Kinney, E.W. Kolb, and M.S. Turner, Phys.Rev.Lett. 79 (1997) 2620.

[25] A. G. Cohen and A. De Rujula, astro-ph/9709132.

[26] A. Milsztajn, T. Lasserre, for the EROS collaboration, astro-ph/0011375.

[27] (The Macho collaboration) C.Alcock, R.A. Allsman, D.R. Alves, et al, astro$\mathrm{ph} / 0011506$.

[28] A.D. Dolgov, K. Griest, work in preparation. 